\title{
PLATAFORMAS DE APRENDIZAJE: ¿HERRAMIENTAS TÉCNICAS O PSICOLÓGICAS?
}

\author{
PLATAFORMAS DE APRENDIZAGEM: \\ FERRAMENTAS TÉCNICAS OU PSICOLÓGICAS? \\ LEARNING PLATFORMS: \\ TECHNICAL OR PSYCHOLOGICAL TOOLS? \\ LES PLATE-FORMES D'APPRENTISSAGE : \\ DES OUTIS TECHNIQUES OU PSYCHOLOGIQUES ?
}

Francisco Sacristán Romero *

\section{RESUMEN}

En el documento se abordan algunos principios de diseño instruccional vinculados con el constructivismo sociocultural y la cognición situada referidos al diseño de entornos de aprendizaje apoyados con tecnologias de la información y comunicación (TIC). Se analizan las potencialidades educativas de dichas TIC en su carácter de signos o instrumentos psicológicos en contraste con su empleo restringido como herramientas técnicas. Se destacan algunas características de los entornos apoyados con las TIC que potencian el aprendizaje y se concluye remarcando la necesidad de realizar investigaciones sobre los mecanismos de influencia educativa y las prácticas educativas reales que ocurren en diversas experiencias escolares y autoiniciadas mediadas por la tecnología.

Palabras-clave: Plataformas de aprendizaje. Instrumentos psicológicos. Tecnologías de la información y comunicación. TIC.

* Doctor en Ciencias de la Información. Profesor e investigador en la Universidad Complutense de Madrid, España (franciscosacristan@ozu.es). 


\section{INTRODUCCIÓN}

\section{"Ni la información es sinónimo de conocimiento ni la recepción de la información garantiza el aprendizaje".}

La creciente diseminación del discurso de la entrada a la sociedad del conocimiento nos enfrenta a una inminente y necesaria reconceptualización e innovación de las teorías de diseño instruccional prevalentes, modeladas en su mayoría en contextos de educación formal, presencial y escolarizada a la vez que fuertemente centrados en el aprendizaje de contenidos disciplinares. En la década pasada hemos atestiguado importantes esfuerzos en el desarrollo de teorías y modelos educativos enfocados a la creación de entornos y comunidades de aprendizaje apoyados con las tecnologías de la información y la comunicación, que han incluido pautas para el diseño, implementación y evaluación de materiales educativos, unidades didácticas, actividades experienciales y objetos de aprendizaje. Por supuesto que este corpus se ha gestado en torno a distintos paradigmas de diseño instruccional y es innegable que en el campo de la psicología de la instrucción, predominan los enfoques constructivistas, en especial los basados en la psicología cognitiva, el enfoque sociocultural y la cognición situada.

No obstante, el término constructivismo parece servir como una especie de "paraguas" para una diversidad de perspectivas (DUFFY; CUNNINGHAM, 2001). Pero a la vez parece existir acuerdo entre dichas perspectivas en que a) el aprendizaje es (o debiese ser) un proceso activo de construcción de significados más que un proceso de adquisición de información y en que b) la instrucción es un proceso de soporte o mediación a dicha construcción que va más allá de la comunicación o transmisión de información acabada. También hay acuerdo, tal como lo planteara en su momento Jerome Bruner, en que el conocimiento no está en el contenido disciplinar, sino en la actividad constructiva (o co-constructiva) de la persona sobre el dominio de contenido tal como ocurre en un contexto socioeducativo determinado.

Pero la metáfora educativa subyacente a los procesos de construcción y al papel de los actores de la educación puede diferir en cuestiones centrales. Rogoff (1994), Hernández (1998), entre otros, han establecido distinciones importantes entre los principales paradigmas psicoeducativos de corte constructivista que derivan en enfoques instruccionales. En tres de las versiones más conocidas de dichos enfoques instruccionales encontramos importantes diferencias:

- En el modelo instruccional de expertos-novatos, se enfatiza la actuación del agente educativo: el experto es quien modela y promueve determinados saberes en el novato).

- En el modelo de descubrimiento individual y colaborativo, resalta la actividad que realiza el aprendiz puesto que los alumnos de forma individual o en grupos pequeños abordan tareas que implican descubrimiento y solución de problemas abiertos.

- En las llamadas comunidades de aprendizaje el acento está puesto en la participación conjunta 
en experiencias socioculturales y colectivas relevantes y auténticas, por lo que se enfatiza el trabajo cooperativo y la co-construcción del conocimiento.

De esta manera, en la enseñanza recíproca y en los diseños instruccionales basados en la metáfora del aprendizaje artesanal o apprenticeship el profesor asume el rol de entrenador (coach o manager) mientras que en la instrucción basada en el aprendizaje basado en problemas $(\mathrm{ABP})$ y en los diseños instruccionales experienciales inspirados en metáfora del aprendiz como científico, el foco de la enseñanza se desplaza a las habilidades de cuestionamiento y prueba sistemática de conjeturas de parte de los estudiantes. Finalmente, si contraponemos la instrucción basada en los modelos cognitivos versus la que se orienta por las premisas de las aproximaciones socioculturales, no sólo nos enfrentamos con la polémica de si la mente (y por ende el aprendizaje) está localizada en la cabeza o en la interacción de los individuos en sociedad, sino vislumbramos diferencias importantes que se establecen en las metas educativas: la reorganización de representaciones y procesos cognitivos frente a los procesos de aculturación en una comunidad de prácticas o el tratamiento instruccional centrado en procesos psicológicos individuales frente a procesos sociales y culturales. Con lo anterior no pretendemos decir que ambas posturas, cognitiva y sociocultural, sean irreconciliables en un proceso de diseño instruccional, sino que el punto de partida en todo diseño implica una toma de postura y la clarificación de la o las perspectivas y metas asumidas. De hecho, desde la década del noventa ha tomado una presencia creciente el enfoque de la cognición situada aplicado al campo del diseño educativo (DÍAZ BARRIGA, 2005). Dicho enfoque, sin desconocer la importancia que tiene el desarrollo de las habilidades cognitivas y del pensamiento, plantea al mismo tiempo que el conocimiento es un fenómeno social, no una "cosa", que es parte y producto de la actividad conjunta de los actores y que se encuentra situado en el contexto y cultura en que se desarrolla y utiliza.

Lo anterior tiene importantes implicaciones instruccionales que abarcan situaciones de enseñanza presencial y mediada por tecnologías. En primer término, puesto que el conocimiento es dependiente del contexto y la cultura, el aprendizaje debe ocurrir en contextos relevantes, en situaciones auténticas, caracterizadas por la cooperación y promover el facultamiento personal y social de los educandos. De esta manera, el conocimiento no es únicamente un estado mental, sino un conjunto de relaciones basadas en experiencias que no tienen sentido fuera del contexto donde ocurren. Otro supuesto importante es que el aprendizaje está mediado por instrumentos, los cuales pueden ser artefactos físicos pero también instrumentos semióticos o signos; como veremos a continuación, las tecnologías de la información y su uso pedagógico pueden explicarse desde esta concepción. 


\section{EL USO DE LAS TIC EN LA INSTRUCCIÓN: ¿HERRAMIENTAS FÍSICAS O PSICOLÓGICAS?}

En el caso del diseño instruccional apoyado con las nuevas tecnologías de la información y la comunicación (TIC) es importante revisar el papel que se otorga a éstas en el proceso educativo. Lo más frecuente es pensar que la introducción de la computadora y los medios en el aula permiten no sólo allanar la distancia geográfica y ampliar la cobertura, sino ante todo suministrar la instrucción de una forma más eficiente y efectiva y se asume que debido a esto se promoverán mejores aprendizajes. Los profesores esperan ante todo que la tecnología les ayude a mostrar a sus alumnos mejores ejemplos de los conceptos y principios que enseñan, oportunidades casi ilimitadas y personalizadas para ejecutar un procedimiento, aprender una técnica o corregir errores, y sobre todo, lograr un ambiente de aprendizaje más entretenido o motivante (DUFFY; CUNNINGHAM, 2001).

No obstante, las llamadas TIC no deben quedarse sólo en el nivel de "herramientas de enseñanza eficaz", en el sentido de dispositivos físicos que ayudan a los alumnos a adquirir y practicar contenidos curriculares de manera más eficiente, sobre todo si el entorno de enseñanza-aprendizaje en su conjunto queda inalterado y no se ha transformado hacia una visión de construcción significativa del conocimiento. Esta es una crítica que se puede hacer a una cantidad considerable de experiencias de enseñanza en línea, donde las TIC están al servicio - eficiente - de modelos de enseñanza transmisivo-receptivos centrados en el aprendizaje declarativo de contenidos estáticos (i.e. lecturas de capítulos o artículos basadas en la resolución de cuestionarios con preguntas literales, ejercicios rutinarios y exámenes objetivos de respuesta cerrada). En este caso, la tecnología se emplea para "amplificar" lo mismo que se ha venido haciendo. Más preocupación aún genera el diseño instruccional que ubica las TIC - el manejo de medios o programas y paquetes informáticos per se - como el fin último de la instrucción (i.e. cuando el aprendizaje de un procesador de textos se centra en el dominio de los comandos e instrucciones propias del programa, no en las competencias comunicativas que vehicula, como serían la expresión de ideas, la exploración de alternativas para comunicar un argumento, la planeación y autorregulación del mensaje comunicado, el destino y sentido social de una composición escrita). De acuerdo con Arends (2004) los profesores pueden utilizar la creciente popularidad que la interacción en línea ha logrado entre los jóvenes tanto para lograr algunas de las metas educativas más tradicionales que existen en torno a la discusiones en clase como para enriquecer dichas discusiones en formas que no era posible en los tiempos pre-Internet. Desafortunadamente, esta segunda opción ha resultado poco explotada al presente.

En las situaciones antes ejemplificadas, las TIC sólo se perciben como herramientas técnicas o artefactos físicos cuyo empleo facilita o hace más eficientes las formas de acción que ocurrirían de otra manera. Sin embargo, se deja de lado el sentido de herramienta semiótica o psicológica propia de la noción de acción mediada planteada en el enfoque vigotskiano. Como es bien sabido, la perspectiva sociocultural inspirada en Vigotsky plantea que el aprendizaje se encuentra mediado por herramientas físicas o 
técnicas y signos o herramientas semióticas (también denominadas instrumentos psicológicos, v. KOZULIN, 2000).

La invención y empleo de una herramienta por los miembros de una comunidad no sólo facilita la acción y aumenta su eficacia, sino que cambia de manera sustancial la forma, estructura y carácter de la actividad. Visto así, las herramientas son "depósitos culturales" (WILSON; MEYERS, 2000) puesto que encarnan la historia de una cultura, ya que proporcionan medios poderosos de transmisión de dicha cultura pero también permiten o limitan el pensamiento y los procesos intelectuales. Así, una herramienta semiótica o instrumento psicológico implica una forma de razonamiento o argumentación asociada a determinadas creencias, reglas y normativas sociales que determinan a su vez el sentido y uso de dicha herramienta.

$\mathrm{Al}$ respecto, podríamos preguntarnos, a manera de ilustración, si el procesador de textos por computadora que hoy empleamos no sólo permite hacer más eficiente o práctica la escritura que hace 25 años. La cuestión sería si el proceso de escritura ha cambiado gracias a esta herramienta, si ha promovido una forma de mediación del funcionamiento cognitivo que antes no existía, si ha influido la forma de trabajar del escritor así como en el producto escrito. Es decir ¿̇ha permitido una forma alternativa de expresar un significado subyacente o podemos hablar de la existencia de un sistema semiautónomo de construcción de significado? (v. DUFFY; CUNNINGHAM, 2001). Otro ejemplo lo encontramos en el hipertexto electrónico, pues de acuerdo con Campos y Medina (2004) es un texto multisecuencial que el lector experimenta como no lineal, en tanto que son posibles múltiples formas de encadenar sus partes y navegar a través del contenido. Aunque puede decirse que las habilidades lectoras convencionales se aplican al interior de cada segmento de texto considerado de forma individual, cuando se va más allá de los fragmentos en sí mismos, la noción previa de unidad del texto se rompe y aparecen reglas y niveles de experiencia diversos. El lector se enfrenta a una forma de textualidad donde los contenidos no sólo son palabras, sino imágenes y sonidos, que se interconectan en trayectorias o encadenamientos abiertos y no únicos, inacabados y susceptibles de elección en función de los intereses, habilidad, experiencia o motivos presentes en la actividad lectora. El eje o principio organizador del contenido así como el centro de atención se desplazan continuamente, y el lector interviene en la toma de decisiones respecto al establecimiento de nuevos vínculos, en la introducción o eliminación de contenidos, en la modificación de la organización y extensión de éstos, de tal forma que hoy en día se habla de hiperlectores y habilidades de hiperlectura (CAMPOS; MEDINA, 2004).

La computadora y en general las llamadas TIC son ejemplos de instrumentos mediacionales que comparten aspectos tanto de herramienta física como semiótica. No obstante, su potencialidad como instrumento mediacional del funcionamiento cognitivo, como sistema de construcción de significados o de transformación y creación de contenidos culturales es menos explotada en el diseño de experiencias educativas en comparación a su atributo como herramienta técnica eficiente. La "novedad" que ofrecen las TIC a profesores y alumnos no son los recursos semióticos aislados que incluyen (lengua 
oral y escrita, lenguajes audiovisual, gráfico o numérico), sino que a partir de la integración de dichos sistemas simbólicos clásicos, se puede eventualmente crear un nuevo entorno de aprendizaje, con condiciones inéditas para operar la información y transformarla. En palabras de César Coll (2004-2005, p. 5):

"No es en las TIC, sino en las actividades que llevan a cabo profesores y estudiantes gracias a las posibilidades de comunicación, intercambio, acceso y procesamiento de la información que les ofrecen las TIC, donde hay que buscar las claves para comprender y valorar el alcance de su impacto en la educación escolar, incluido su eventual impacto sobre la mejora de los resultados del aprendizaje”.

\section{ENTORNOS DE APRENDIZAJEY POTENCIALIDAD DE LAS TIC}

Ahora bien, las herramientas informáticas, en su calidad de medios para introducir y manipular tanto las ideas como los recursos, pueden emplearse con fines de tratamiento, de búsqueda, de recopilación, de organización o de creación de la información (HANNAFIN; LAND; OLIVER, 2000). Es decir, el agente educativo o los mismos estudiantes pueden tener en mente distintas finalidades cuando hacen uso de las tecnologías: buscar información, contrastarla, organizarla, compartirla, asegurar cierto tipo de comunicación entre profesor y alumnos o entre pares, hacer posible el trabajo en equipo, etc. y el acento puesto en cada una de ellas puede cambiar el sentido y las prácticas en un entorno de aprendizaje dado.

La preocupación de connotados autores en el campo del diseño instruccional estriba en la elaboración de teorías del diseño educativo orientadas hacia la promoción de actividades cognitivas e interactivas más potentes, así como a la reorganización y extensión de los aprendizajes, en un sentido de construcción activa del conocimiento, tanto en el plano intra como en el intermental. Así, uno de los principales retos de la instrucción apoyada con TIC es ofrecer nuevas representaciones y perspectivas de distintos fenómenos, de interés tanto científico como cotidiano, que de otra manera no sería posible desarrollar, y de esta manera, contribuir a transformar tanto nuestra comprensión y prácticas como la cultura misma.

En este punto es importante una precisión respecto al concepto de entorno o ambiente de aprendizaje. Éste se refiere a un determinado estilo de relación entre los actores que participan en el contexto de un evento determinado, con una serie de reglas que determinan la forma en que se organizan y participan e incluye una diversidad de instrumentos o artefactos disponibles para lograr unos fines propuestos. Si alguno de estos elementos cambia o falla, el ambiente se transforma. Como podemos ver, el concepto no sólo abarca el espacio físico o los medios disponibles, sino aquellos elementos básicos que definen el diseño instruccional de toda propuesta pedagógica (apoyada o no con TIC): "el espacio, el estudiante, el asesor, los contenidos educativos y los medios" siendo "la estrategia didáctica la que permite una determinada dinámica de relación entre los 
componentes educativos" (DIRECCIÓN DE INVESTIGACIÓN Y COMUNICACIÓN EDUCATIVAS DEL ILCE, 1999, p. 57). Por lo anterior resulta evidente que puede existir una gran variabilidad en el diseño de entornos de aprendizaje apoyados por TIC y que las tecnologías y los medios pueden servir diferencialmente para muchos propósitos.

Como resultado de una amplia revisión de las características más sobresalientes de los entornos de aprendizaje apoyados por las TIC y teniendo como marco de referencia la aproximación constructivista de los procesos de enseñanza y aprendizaje, Coll (20042005, p. 10) plantea las potencialidades de dichas tecnologías en el diseño educativo. Este autor destaca las características de interactividad, multimedia e hipermedia como las que más potencian a las TIC como instrumentos psicológicos mediadores de las relaciones entre los alumnos y los contenidos, mientras que la conectividad potencia las relaciones entre los actores (v. Cuadro 1). Estas características tienen que ver con las posibilidades de acceso a la información, a la manera de representarla y a las posibilidades de interacción. Desde la perspectiva del diseño de un entorno de aprendizaje, inciden en las relaciones entre agentes educativos, alumnos y contenidos y son susceptibles de establecer nuevas formas de mediación.

\section{Cuadro I. Características de los entornos simbólicos basados en las TIC y sus potenciali- dades para el aprendizaje. Fuente: Coll (2004-2005).}

\begin{tabular}{|l|l|}
\hline Formalismo & $\begin{array}{l}\text { Implica previsión y planificación de las acciones. Favorece la toma de conciencia y la } \\
\text { autorregulación. }\end{array}$ \\
\hline Interactividad & $\begin{array}{l}\text { Posibilidades que ofrecen las TIC de que el estudiante establezca una relación contingente } \\
\text { e inmediata entre la información y sus propias acciones de búsqueda y procesamiento. } \\
\text { Permite una relación más activa y contingente con la información. Potencia el protago- } \\
\text { nismo del aprendiz. Facilita la adaptación a distintos ritmos de aprendizaje. Tiene efectos } \\
\text { positivos para la motivación y la autoestima. }\end{array}$ \\
\hline Dinamismo & $\begin{array}{l}\text { Ayuda a trabajar con simulaciones de situaciones reales. Permite interactuar con realidades } \\
\text { virtuales. Favorece la exploración y la experimentación. }\end{array}$ \\
\hline Multimedia & $\begin{array}{l}\text { Capacidad de los entornos basados en TIC para combinar e integrar diversas tecnologías. } \\
\text { Permite la integración, la complementariedad y el tránsito entre diferentes sistemas y formatos } \\
\text { de representación (lengua oral y escrita, imágenes, lenguaje matemático, sonido, sistemas } \\
\text { gráficos, etc.). Facilita la generalización del aprendizaje. }\end{array}$ \\
\hline Conectividad & $\begin{array}{l}\text { Resultado de la convergencia de la naturaleza multimedia del entorno más la utilización } \\
\text { de una lógica hipertextual. Comporta la posibilidad de establecer formas diversas y flexibles } \\
\text { de organización de las informaciones, estableciendo relaciones múltiples y diversas entre } \\
\text { ellas. Facilita la autonomía, la exploración y la indagación. Potencia el protagonismo del } \\
\text { aprendiz. }\end{array}$ \\
\hline $\begin{array}{l}\text { Permite el trabajo en red de agentes educativos y aprendices. Abre nuevas posibilidades al } \\
\text { trabajo grupal y colaborativo. Facilita la diversificación, en cantidad y calidad, de las ayu- } \\
\text { das que los agentes educativos ofrecen a los aprendices. }\end{array}$ \\
\hline
\end{tabular}


Desde nuestra perspectiva, tomando como referente las citadas características y en función de las finalidades y usos que se den a las TIC, se podrán conformar entornos de aprendizaje centrados en el alumno, individualizados y significativos en la medida en que se adapten a las necesidades y motivos, estilos de aprendizaje, capacidades y conocimientos previos de los destinatarios.

Pero al mismo tiempo, tomando como referente las premisas del abordaje sociocultural y situado que hemos mencionado antes, a nuestro juicio lo más importante asegurar la situatividad del entorno de aprendizaje, en cuanto éste permita al alumno interactuar con situaciones reales o auténticas, resolver problemas relevantes, aprender a tomar decisiones en situaciones que le plantean el reto de la incertidumbre o el conflicto de valores, es decir, adquirir los saberes y habilidades propios de la comunidad de práctica social o profesional a que pertenece o se pretende integrar. Al respecto, Hung y Der-Thanq (2001), con base en el pensamiento vigotskiano y los principios de la cognición situada antes expuestos, proponen que el diseño instruccional en una comunidad de práctica en línea o de aprendizaje basado en la Web se sustenta en cuatro dimensiones:

- Situatividad: Se fomenta mediante actividades contextualizadas, como tareas y proyectos auténticos, basados en necesidades y demandas reales, tomando en cuenta el conocimiento explícito e implícito sobre el asunto en cuestión (creencias, normas del grupo).

- Comunalidad: Se fomenta en la medida en que hay intereses y problemas compartidos entre los integrantes de la comunidad, lo que permite el establecimiento de metas compartidas.

- Interdependencia: Ocurre en la medida en que los participantes varíen en el nivel de competencia o expertez, es decir, si hay diferencias en conocimiento, habilidades, perspectivas, opiniones y necesidades, y siempre y cuando se logren entablar relaciones de beneficio mutuo (por ejemplo, los novatos obtienen apoyo y respuestas de los expertos y éstos ganan reputación en el campo, partiendo de la idea que completar una tarea no será posible si se maneja de manera individual).

- Infraestructura: Implica la existencia de reglas o sistemas que promueven la motivación y participación, una serie de mecanismos de rendición de cuentas de los participantes y la disposición de estructuras de facilitación de la información y la interdependencia.

Nótese que dichas dimensiones guardan cierta relación con las propuestas por Coll, aun cuando en este caso, los autores centran su propuesta en el modelo de comunidades de práctica, donde los participantes se encuentran embebidos de manera colectiva en situaciones auténticas, donde ocurren actos sociales constructivos (más bien co-constructivos) que tienen sentido en contextos de aplicación y uso determinados. En una comunidad de práctica se comparten conocimientos explícitos e implícitos, lenguajes, géneros, herramientas y signos que les son propios, sucediendo que los miembros pueden participar a diferentes niveles, según sus motivos y nivel de competencia. La filosofía de estas comunidades en línea plantea que el conocimiento reside en las personas, no en las 
bases de datos. Así planteado, lo valioso de un sistema en línea es la posibilidad de manejar y facilitar una interacción intensa y constructiva entre los participantes, con miras a lograr el mayor dinamismo posible entre el flujo de la información (contenidos y recursos) y la participación de los integrantes de la comunidad.

No obstante, hay que recalcar el sentido de "potencial" de las características y dimensiones antes mencionadas, en el sentido de que su inclusión por sí sola no garantiza el logro de aprendizajes significativos o la promoción de habilidades complejas de pensamiento. De hecho, este es uno de los aspectos abiertos al debate, sobre todo considerando el protagonismo y autonomía que se otorga al aprendiz. Retomando un ejemplo anterior, puede suceder que el lector de un hipertexto que no posee las habilidades de hiperlectura referidas, ante la carencia de una lógica secuencial y del patrón lineal de lectura que le es conocido, sea incapaz de organizar e integrar los fragmentos de información por los que navega alternativamente y su aprendizaje sea repetitivo y poco crítico. De ahí la necesidad de educar a los alumnos en las habilidades de indagación y exploración autónoma requeridas en este tipo de entornos y de sentar las bases del trabajo colaborativo y estratégico que éstos demandan.

Algunos principios de enseñanza-aprendizaje con implicaciones para el diseño de la instrucción en entornos apoyados por TIC, congruentes con lo que hemos venido exponiendo, se mencionan a continuación:

- El aprendizaje es un proceso de construcción de conocimiento y la enseñanza una ayuda asistida o mediada a dicho proceso.

- Se pueden construir muchas visiones en torno a campos de conocimiento determinados, la instrucción debe permitir múltiples perspectivas.

- El conocimiento es dependiente del contexto, por lo que el aprendizaje debe ocurrir en contextos relevantes.

- El aprendizaje se encuentra mediado por herramientas y signos y en el proceso participan diversos agentes educativos.

- El aprendizaje es una actividad social y dialógica.

- La cognición es situada, es parte y producto de la actividad, del contexto y de la cultura en que se desarrolla y utiliza.

- La cognición se distribuye socialmente: el aprendizaje no sólo es internalización del conocimiento, sino ante todo, transformación de la participación de las personas en una comunidad social.

- La importancia de los procesos de toma de conciencia de lo que se ha aprendido y se sabe, así como de los procesos de práctica reflexiva y el desarrollo de estrategias para el aprendizaje deben colocarse como una de las principales metas de un sistema instruccional.

Es innegable que la incertidumbre, el cambio y la complejidad rigen actualmente nuestros sistemas educativos y no es fácil prever la dirección que tomarán los modelos educativos o las reformas curriculares en el mediano plazo. No obstante, con base en la 
exposición que hemos venido desarrollando, podemos plantear algunos elementos que caracterizarán el diseño de entornos de aprendizaje apoyados con TIC.

En primera instancia, a nuestro juicio es indispensable contar con diseños flexibles, orientados o centrados en el alumno, no en la transmisión de la información. El punto focal del diseño didáctico será la previsión de interacciones constructivas tomando en cuenta los elementos del triángulo didáctico: los agentes educativos, los usuarios del sistema y los contenidos o saberes culturales sobre los que se opera, considerando las posibilidades y restricciones de los instrumentos semióticos incluidos en un sistema instruccional determinado. Consideramos que la tendencia apunta hacia el diseño de entornos de aprendizaje donde se trabaje en modalidades híbridas o mixtas, donde se intercalan episodios de enseñanza grupal presencial con tutoría individualizada y en grupos pequeños, trabajo cooperativo para el debate y construcción conjunta del conocimiento y la generación de todo tipo de producciones innovadoras, en conjunción con interacciones virtuales o a distancia.

Se plantea como un desarrollo necesario el incremento de la participación entre estudiantes y grupos de diversas instituciones y disciplinas en tareas complejas y compartidas, centradas en la toma de decisiones, la solución de problemas y la intervención en la práctica. Esto implica que nuestras actuales definiciones de educación presencial, abierta y distancia deberán replantearse. De acuerdo con Reigeluth (2000), el paradigma actual de la enseñanza se basa aún en la estandarización y se tiene que dar un cambio importante hacia la formación de personas que tomen iniciativas y hagan honor a la diversidad. Por otra parte, los enfoques y estrategias provenientes de la enseñanza situada, con sustento en el paradigma sociocultural, la cognición situada y el cognitive apprenticeship, están impactando sustancialmente el diseño educativo, tanto a nivel del currículo como de la instrucción y hacia allá apunta el diseño de entornos de aprendizaje tanto presenciales como virtuales (DÍAZ BARRIGA, 2003). Por lo anterior, éstos y otros autores (HANNAFIN; LAND; OLIVER, 2000) plantean los rasgos deseables en un nuevo paradigma de diseño educativo, acorde a la sociedad de la información:

- Se dejará a los alumnos una fuerte iniciativa (aprendizaje autodirigido y fomento de la autonomía, desarrollo de la agencia y la autorregulación) por lo cual se incorporarán modelos y estrategias de educación facultadora y para la vida.

- Se trabajará sobre todo en equipos cooperativos sobre tareas reales, de la vida cotidiana o de un ámbito de competencia profesional determinado en contacto estrecho con usuarios y en escenarios reales afrontando experiencias prácticas, concretas y realistas (formación en la práctica, in situ).

- El currículo y la enseñanza se organizarán en entornos de aprendizaje abiertos, donde se fomente el razonamiento divergente y las perspectivas múltiples (no la perspectiva correcta); los alumnos deben poder escoger (y proponer) entre una variedad de métodos y actividades.

- Permitirá utilizar el potencial de herramienta cognitiva (o instrumento semiótico) de las tecnologías más avanzadas. 
- Se adaptará a ritmos personalizados, trayectos flexibles y alternativos; los alumnos podrán trabajar en una experiencia educativa hasta que alcancen unos niveles adecuados de desempeño.

- Se privilegiarán tareas cognitivas complejas y de relevancia social, necesarias para solucionar problemas en campos complejos, cambiantes, inciertos.

- Se requiere de sistemas instruccionales que estén en constante diálogo con el alumno y que puedan actualizar continuamente la información sobre sus progresos, desempeño, actitudes y expectativas.

- La evaluación (en congruencia con la enseñanza) abarcará el saber, el saber hacer y el ser; se centrará en el desempeño y competencias adquiridas, en la valoración de tareas generativas y en el seguimiento de procesos y mecanismos de autorregulación. Será importante explorar no sólo qué información declarativa se ha adquirido, sino qué habilidades específicas y disposiciones o actitudes se manifiestan en la forma de competencias sociofuncionales complejas.

- Se dará una fuerte expansión de sistemas instruccionales en áreas no sólo cognoscitivas y disciplinares, sino en desarrollo emocional, del carácter (personal-moral) y social.

Un aspecto a resaltar es que se plantea que los diseñadores de la instrucción no serán únicamente los docentes o los expertos en diseño educativo y tecnologías, sino los propios alumnos-usuarios. Al respecto, Reigeluth (2000) apunta que una buena parte de lo diseñado debería estar hecho por los propios alumnos (diseñadores-usuarios) mientras están aprendiendo, con la ayuda de un programa informático que elabore opciones basadas en la información recogida por los mismos alumnos; esto quiere decir que el alumno podrá solicitar al sistema determinados métodos educativos y éste podrá a su vez aconsejar o tomar decisiones sobre su conveniencia.

\section{A MANERA DE CIERRE: LA IMPORTANCIAY RETOS DE LA INVESTIGACIÓN EDUCATIVA EN ENTORNOS APOYADOS CON TIC}

Con base en lo expuesto anteriormente, podemos decir que aunque actualmente en el diseño de la instrucción, tanto en entornos presenciales como a distancia, se están incorporando de manera creciente las TIC, existe una gran diversidad en el sentido y usos de las tecnologías. Desde una perspectiva constructivista, sobre todo de corte sociocultural, tal incorporación requiere enfocarse en la creación de ambientes de aprendizaje que propicien la participación de los actores en actividades de valor innegable para los individuos y sus grupos o comunidades de pertenencia. La conformación de un nuevo paradigma educativo como el que aquí se ha descrito, requiere de la incorporación de enfoques como el de la enseñanza situada (DÍAZ BARRIGA, 2003, 2005). Puesto que las personas actúan y construyen significados dentro de sus comunidades de práctica y todo pensamiento, aprendizaje y cognición se encuentran situados dentro de contextos 
socioeducativos y culturales particulares, tanto el diseño como el desarrollo y evaluación de la instrucción están adoptando crecientemente los principios de la cognición y enseñanza situada. Al respecto, Wilson y Meyers (2000) plantean que el punto focal del diseño de la instrucción se ubica hoy en día la conformación de ambientes de aprendizaje flexibles, orientados al facultamiento y a la participación activa en comunidades de práctica, con una fuerte apertura a una gran diversidad de roles e identidades de parte de los actores y donde existe una profusa mediación de artefactos, a los cuales se busca dar un uso inteligente y propositivo.

Para concluir, respecto a si sabemos realmente cómo intervienen las TIC en la construcción del conocimiento, habría que reconocer que falta todavía mucha investigación al respecto e incluso desarrollar metodologías de investigación apropiadas para indagar cómo operan los mecanismos de influencia educativa cuando la mediación del aprendizaje ocurre a través de dichas tecnologías. Hay investigación acerca de actitudes y opiniones respecto a su incorporación, sobre el nivel de alfabetización tecnológica y su frecuencia de empleo, sobre la disposición que generan en alumnos y profesores, sobre el tipo de dispositivos tecnológicos empleados o bien las comparaciones clásicas de rendimiento académico entre educación con y sin TIC, entre educación presencial y a distancia. Este corpus de investigación es importante, aunque dada la multideterminación del fenómeno educativo y debido a la diversidad de contextos educativos, de oportunidades y restricciones en los mismos, los resultados en ocasiones son contradictorios. Un ejemplo de lo anterior es el metanálisis conducido por Bernard y Abrami (2004) con 232 investigaciones realizadas entre 1985 y 2002 donde se comparan experiencias de educación a distancia versus instrucción presencial en salón de clases, considerando aspectos como el aprovechamiento académico, la actitud y la retención de contenidos. En este caso, resalta la heterogeneidad de los resultados, puesto que en muchos estudios la educación a distancia supera a su contraparte en aula en las variables mencionadas, pero en otros tantos, arroja resultados mucho más pobres.

Consideramos que falta conducir estudios sobre procesos de interacción e influencia educativa en distintos tipos de entornos de aprendizaje apoyados con las tecnologías y analizar, por ejemplo, las formas de organización social y participación, los procesos de construcción de significados que se propician, los mecanismos de mediación empleados por los agentes educativos y propiciados por las propias tecnologías y medios empleados, la estructura de las tareas académicas, la naturaleza de los contenidos que se enseñan y lo que en realidad se aprende, su sentido y funcionalidad.

Otra línea de investigación muy importante se refiere a las actividades constructivas de los alumnos, como serían el estudio de los procesos de aprendizaje, motivacionales y de autorregulación desplegados por éstos en su interacción con las TIC. Algunos estudios recientes arrojan luz al respecto, como sería el caso del del Rogers y Swan (2004) acerca del aprendizaje autorregulado y la búsqueda en Internet en alumnos universitarios. Estos investigadores encuentran que no todos los alumnos realizan las búsquedas en Internet de la misma manera, que requieren apoyos diferenciados en el proceso y que los alumnos se involucran en este tipo de tareas con mayor o menor éxito en función de su motivación y de las habilidades que poseen. Asimismo, destaca la importancia de las estrategias 
autorregulatorias de los estudiantes, tanto para la adquisición como para la transformación de la información, destacando los componentes atencionales, de selectividad, conectividad, planificación de tareas y rutinas y monitoreo continuo.

Por otra parte, no sólo habrá que investigar qué sucede en los entornos de educación formal escolarizada; aprenderíamos mucho de las posibilidades de empleo de las tecnologías para promover la construcción social del conocimiento, la motivación, la creatividad, si se investigara con una mirada psicopedagógica lo que ocurre en experiencias no formales, no escolarizadas, más bien autoiniciadas y de interacción libre a través de los medios y las tecnologías informáticas. Por ejemplo, el estudio de los procesos e interacciones que ocurren en torno a lo que hacen los jóvenes en espacios de tiempo libre y recreación y no sólo me refiero a chats o foros libres y eventuales, sino a la constitución de verdaderas redes y comunidades de intercambio y construcción de conocimiento. Hace falta comprender los mecanismos cognitivos, afectivos, de interacción social e influencia educativa que entran en juego cuando niños y jóvenes, incluso adultos, están frente a los videojuegos, el Internet o la televisión interactiva. Finalmente, otro aspecto clave a estudiar es el contexto educativo donde se introducen las innovaciones tecnológicas, para así analizar de qué depende su empleo exitoso, las posibilidades y restricciones que permite el entorno de aprendizaje como un sistema de prácticas socioculturales.

\section{Notas}

1. De acuerdo con Berger y Kam (1996) del Laboratorio de Investigación Aplicada de Penn State University, el diseño instruccional es el proceso sistemático que conduce a la creación de sistemas instruccionales, mientras que el desarrollo instruccional es el proceso de implementar dicho sistema o plan e incluye su evaluación y mantenimiento. En este trabajo asumimos que ambos procesos deberían ser indisociables y que el diseño y desarrollo sistemático de las especificaciones psicopedagógicas contenidas en un sistema instruccional requiere, entre otras cosas, tanto una toma de postura como un sustento sólido y congruente en las teorías del aprendizaje y la enseñanza así como en la investigación de la práctica educativa que ocurre en las aulas.

2. Las tecnologías de la información y comunicación no son equiparables sólo a las tecnologías informáticas desarrolladas a partir de la segunda mitad del siglo XX, aunque hoy en día es el sentido más empleado del término y el que adoptan los diseñadores instruccionales actuales. Para hacer esta distinción, en algunos casos se suele hablar de "nuevas" tecnologías de la información y comunicación (NTIC). Las TIC como instrumentos creados por el ser humano para representar, transmitir y recrear el conocimiento, han pasado por diferentes etapas de desarrollo, siendo algunos de sus hitos más reconocidos la creación del lenguaje oral, la escritura, la imprenta, el teléfono, etc., puesto que han propiciado nuevas maneras de vivir y trabajar juntos.

3. La semiótica (del griego semeion, signo) también llamada semiología, es la ciencia que estudia los sistemas de signos dentro de una sociedad, es decir, nos permite analizar nuestros recursos y acciones sociales para comunicar significados. El lenguaje es por supuesto nuestro principal recurso semiótico, pero también lo son la notación algebraica, los diagramas y mapas, la notación musical, entre otros. 


\section{Referências}

ARENDS, Richard. Learning to teach. 6. ed. New York: McGraw Hill, 2004.

BERGER, Carl; KAM, Rosalind. Training and instructional design. Laboratorio de Investigación Aplicada, Penn State University, 1996.

BERNARD, Robert; ABRAMI, Philip. How does distance education compare with classroom instruction? A meta-analysis of the empirical literature. Review of Educational Research, v. 74, n. 3, p. 379-439, 2004. CAMPOS, Antonio; MEDINA, Lucía. Hipertexto, lectura e Internet: Resultados de un estudio en el nivel medio superior. Tecnología y Comunicación Educativas, v. 18, n. 39, p. 32-44, enero-junio 2004. COLL, César. Psicología de la educación y prácticas educativas mediadas por las tecnologías de la información y la comunicación: una mirada constructivista. Sinéctica, n. 25, Sección Separata, p. 1-24, agosto 2004-enero 2005.

DÍAZ BARRIGA, Frida. Cognición situada y estrategias para el aprendizaje significativo. Revista Electrónica de Investigación Educativa, v. 5, n. 2, 2003. http://redie.ens.uabc.mx/vol5no2/contenido-arceo.html

DÍAZ BARRIGA, Frida. Enseñanza situada: vínculo entre la escuela y la vida. México: McGraw Hill, 2005.

DIRECCIÓN DE INVESTIGACIÓN Y COMUNICACIÓN EDUCATIVAS (ILCE). Diseño de ambientes de aprendizaje. Tecnología y Comunicación Educativas, v. 13, n. 29, p. 55-58, enero-junio 1999. DUFFY, Thomas; CUNNINGHAM, Donald. Constructivism: implications for the design and delivery of instruction. The Handbook of Research for Educational Communications and Tecnology, 2001. http://www.aect. Org/Intranet/Publications/edtech/07/index.html. Recuperado el 16 nov. 2001. HANNAFIN, Michael; LAND, Susan; OLIVER, Kevin. Entornos de aprendizaje abiertos: fundamentos, métodos y modelos. En: REIGELUTH, Charles (Ed.). Diseño de la instrucción: teorías y modelos. Madrid: Aula XXI Santillana, Parte I, p. 125-152, 2000.

HERNÁNDEZ, Gerardo. Paradigmas en psicología de la educación. México: Paidós, 1998.

HUNG, David; DER-THANQ, Chen. Situated cognition, Vygotskian thought and learning from communities of practice perspective: implications for the design of Web-based E-learning. Education Media International, 2001. http://www.tandf.co.uk/journals. Recuperado el 9 ago. 2005.

KOZULIN, Alex. Instrumentos psicológicos: la educación desde una perspectiva sociocultural. Barcelona: Paidós, 2000.

REIGELUTH, Charles. ¿En qué consiste la teoría de diseño educativo y cómo se está transformando? En: Diseño de la instrucción: teorías y modelos. Madrid: Aula XXI Santillana, Parte I, p. 15-40, 2000.

ROGERS, Donna; SWAN, Karen. Self-regulated learning and Internet searching. Teachers College Record, v. 106, n. 9, p. 1.804-1.824, 2004. http://.tcrecorf.org ID Number 11671. Recuperado el 9 ago. 2005. ROGOFF, Barbara. Developing understanding of the idea of communities of learners. Mind, Culture and Activity, v. 1, p. 209-229, 1994.

WILSON, Brent; MYERS, Karen. Situated cognition in theoretical and practical context. En: JONASSEN, David; LAND, Susan (Eds.). Theoretical foundations of learning environments. Mahwah, New Jersey: Lawrence Erlbaum, cap. 3, p. 57-88, 2000. 


\section{Plataformas de aprendizagem: ferramentas técnicas ou psicológicas?}

\section{Resumo}

Neste estudo abordam-se alguns princípios de desenho instrucional vinculados ao construtivismo sóciocultural e à cognição situada com relação ao desenho de ambientes de aprendizagem apoiados em tecnologias da informação e comunicação (TIC). Analisam-se as potencialidades educativas dessas tecnologias em seu caráter de signos ou instrumentos psicológicos, em oposição ao seu emprego restrito como ferramentas técnicas. Destacam-se algumas características dos ambientes apoiados com as TIC que potencializam a aprendizagem e se conclui enfatizando a necessidade de conduzir investigaçôes sobre os mecanismos de influência educativa e as práticas educativas reais que ocorrem nas diversas experiências escolares e auto-iniciadas mediadas pela tecnologia.

Palavras-chave: Plataformas de aprendizagem. Instrumentos psicológicos. Tecnologias da informação e comunicação. TIC.

\section{Learning platforms: technical or psychological tools?}

\section{Abstract}

In this document, some principles for instructional designing related to socio-cultural constructivism and the situated cognition, referring to the design of learning patterns based on information and communication technologies (ICT) are dealt with. The educative potencialities of those technologies are analyzed as to their sign character or psychological instruments in contrast with their restricted use as technical tools. A few characteristics of the background support of the ICT which potentialize learning are pointed out. Some conclusions are reached making clear the need to conduct research on the mechanisms of educational influence and the real educational practices which occur in various school experiences, autoinitiated and mediated by technology.

Keywords: Learning platforms. Psychological instruments. Information and communication technology. ICT.

\section{Les plate-formes d'apprentissage : des outis techniques ou psychologiques?}

Résumé

Dans ce travail, on aborde des principes pour le dessein instructionnel relationné au constructivisme socio-culturel et la cognition située, en référence au dessein de contours d'apprentissage basés sur les technologies de l'information et de la communication (TIC). Le potentiel éducatif de ces technologies est analysé quant au charactère de signe ou instrument psychologique qui contrastent avec leur utilisation restricte comme outis techniques. Quelques charactéristiques de l'appui de fond de ces TIC qui potentialisent l'apprentissage sont mises en relief. Des conclusions sont tirées quant au besoin de faire de la recherche sur les méchanismes de l'influence éducationnelle et les vraies pratiques éducatives qui ont lieu dans les multiples expériences scolaires, autoinitiées et médiées para la technologie.

Mots clefs: Plate-formes d'apprentissage. Instrument psychologique. Technologie de l'information et de la communication. TIC.

Recebido: 05.05 .2006

Aceito: 12.06.2006 
